\title{
Awareness without explicit recall. Features and research perspective
}

\author{
Marco Cascella ${ }^{* *}$ and Maria Rosaria Muzio ${ }^{2}$ \\ ${ }^{1}$ Department of Anesthesia and Pain Medicine, National Cancer Institute-IRCCS-G. Pascale Foundation, Italy \\ ${ }^{2}$ Division of Infantile Neuropsychiatry, UOMI-Maternal and Infant Health, Italy
}

\begin{abstract}
Intraoperative awareness (IA) is the postoperative recollection of an unexpected and unwanted experience occurred during general anesthesia. It is a serious and rare anesthesia complication, with a complex physiopathology, and clinical features often poorly understood. Anesthesiologists are led to believe that all the patients who suffered from an IA event can report their experience through a process of explicit memory retrieving. The axiom that explains the correlation between IA occurrence and conscious recollection (IfI do not remember it, I have not experienced it') is only in part true, and applicable to the anesthesia awareness with explicit recall (AAWR) phenomenon, which represents the best-known IA subtype. It is underestimated that anesthesia practice may interfere with the hidden subconscious world. Indeed, an intraoperative unexpected sensory experience can more often shift the explicit memorization way and, in turn, configures the awareness without explicit recall (AWER) picture (the other IA subtype). It is debated if these episodes may be defined as conscious experiences without subsequent recollection on recovery, or they really represent the expression of unconscious experiences. Probably, both hypotheses could be accepted. Many dark sides should be better explored, but it is certain that this underestimated complication is strictly closed to the development of postoperative psychological/psychiatric sequelae. The present work is aimed to give an overview on the AWER phenomenon, suggesting strategies for studying these potentially deleterious 'experiences'.
\end{abstract}

\section{The paradox of intraoperative awareness}

Anesthesia awareness with recall during general anesthesia (AAWR) is the explicit recall of sensory perceptions of the patient during anesthesia [1,2]. Specifically, these unexpected and unwanted experiences may regard hearing sounds, pain, and immobility, sometimes together with extreme anxiety and distress because of the inability to communicate (e.g., due to paralysis induced by neuromuscular blocking agents) [3]. The recalling of these experiences can be reported at the emergence from anesthesia, in the recovery room, or several days/months later. Thus, intraoperative awareness (IA) is a well distinct phenomenon from the anesthetic/intraoperative dreaming which represents a paraphysiological event in course of anesthesia and sedation [4]. On the contrary, IA is a true, but rare anesthesia complication with an overall incidence of approximately 1 every 19,000 anesthetics [5]. Investigations in this field are usually prospectively performed by recording - and subsequently by studying - spontaneous patients' reports at the end of surgery, or later. On the other hand, structured interviews, such as the Brice's questionnaire [6], are often preferred because the absence of a direct interview should underestimate the real incidence of that complication [7]. Again, as Sebel et al. [8] stated, a wide range of subjects may not choose to report their experience unless they are asked directly about it, even in more than 1 occasion ('Don't ask, don't tell'). A retrospective analysis finalized to detect AAWR cases seems to be a very difficult challenge. This approach is often inaccurate and requires a careful collection of information. Nevertheless, these serious limitations should be in part filled with an ad hoc strategy, for instance through the analysis of data obtained for a postoperative psychological assessment which is often performed as routine, in the oncological context. In a previous study conducted on cancer patients, we followed this strategy and detected an AAWR incidence of 1:10,550 [9].
On these premises, AAWR is a paradox of anesthesia as the aim of general anesthesia is to obtain unconsciousness and amnesia. However, because AAWR occurs in extremely rare cases, it should be easily relegated to secondary importance. The matter is more complicated. There are, indeed, two significant issues to be considered. The former regards the potential clinical impact of that complication (i.e., psychological awareness consequences), whereas the latter is closed to clinical and psychological features of a special type of intraoperative awakening which differs from the classical AAWR phenomenon. About the awareness consequences, the intraoperative unexpected awakening can trigger a wide range of psychological sequelae, including post-traumatic stress disorder (PTSD). Classically, PTSD is a serious condition which develops following a traumatic event. Patients who suffered from AAWR have been described as recalling fragments of their surgery in nightmares and flashbacks in which they re-experience paralysis, suffocation, pain, or conversations between surgical personnel. Consequently, these patients usually avoid hospitals, doctors and television programs with hospital themes. Commonly reported hyperarousal symptoms include easy startle, hypervigilance, and irritability [10].

*Correspondence to: Marco Cascella, Department of Anesthesia and Pain Medicine, National Cancer Institute-IRCCS-G. Pascale Foundation, Via Mariano Semmola, Naples, 80131, Italy, Tel: + 39081 5903586, Fax: + 39081 5903778, E-mail: m.cascella@istitutotumori.na.it

Key words: anesthesia awareness, memory, memory consolidation, general anesthetics

Received: March 06, 2018; Accepted: March 19, 2018; Published: March 23, 2018 


\section{The false axiom: 'If I do not remember it, I have not ex- perienced it'}

Usually, anesthesiologists are led to believe that all the patients who suffered from an IA event are able to report their experience through a process of explicit memory ('If I do not remember it, I have not experienced it'). According to the Myles' definition, IA is 'the postoperative recollection of events occurring during general anesthesia' [11]. The term 'recollection' refers to 'a memory of something, or the ability to remember past events' [12]. Thus, is not necessarily related to an explicit-spontaneous, or induced-report.

In neurobiological terms, each IA episode represents a combination of a sensory perception with a complex memorization process which, in turn, leads to consolidation [13]. Different memory models have been proposed, but their description falls out of the scope of this work (for more details see $[14,15]$ ). However, we generally assume that the memory consolidation process is, in part, beyond conscious control and therefore does not necessarily imply that during anesthesia the awakening patient opens his/her eyes or can interact with personnel in the theatre. The neurophysiological background is the linkage between consciousness and memory. It is possible that a patient under anesthetic action may be subjected to an unconscious memory formation. Consequently, the subject is unable to realize a report as the consolidation process shifted the explicit memorization way [16]. This picture configures the awareness without explicit recall (AWER) phenomenon, which represents the other IA subtype. This subliminal learning during anesthesia is a complex phenomenon involving many neurophysiological factors, yet to be elucidated [17]. In this lack, we are aware that the mechanism of anesthesia-related unconscious memory is very efficient as Sanders et al. [18] demonstrated that the incidence of AWER was significantly higher than that of IA with a conscious recall.

What is the destiny of this unexpected experience? Like other types of unconscious/subliminal processes, any AWER episode may remain in the long-term memory system but may be resumed in terms of nightmares and flashbacks. In this circumstance, the subject re-experiences paralysis, suffocation, pain, or conversations of the surgery stuff. Reports of hyperarousal symptoms such as easy startle, hypervigilance, and irritability are common. Although the occurrence of AWER does not seem to entail significant early changes on postoperative psychological function, implicit memory is a memory of which one is not consciously aware but can nevertheless impact performance and behavior. Moreover, it has been emphasized that this underestimated complication is strictly closed to the development of postoperative psychological/psychiatric sequelae, not necessarily expressed as PTSD, but often manifested as other syndromal or subsyndromal mental disorders, such as acute stress disorder, and subsyndromal PTSD. For instance, Samuelsson et al. [19] reported a higher incidence of late psychological symptoms (e.g., sleep disturbances, nightmares, daytime anxiety, and fear about future anesthesia) after awareness, up to $84 \%$. To complicate the picture, the incidence of AWER has been indicated to be significantly higher than the incidence of AAWR [20]. Given these premises, we can assume that AWER is a very insidious long-term anesthesia complication.

\section{Perspective on research about the awareness without explicit recall phenomenon}

Consequently, studying the destiny of these intraoperative not recalled 'experiences', and modality of investigation in this complex field, could represent an interesting subject-matter research as well as a topic connected across different disciplines, such as anesthesiology, neurology, psychiatry, and psychology. The lack of studies might be due to methodological issues. It may reflect, indeed, the lack of an exhaustive model for studying this fascinating phenomenon as well as the prejudice that all these bits of unconscious experiences fall into oblivion. However, it is well-known that consolidation regards also implicit memory and varies with factors such as the timing of stimuli exposure [21], type of information to be learned, and age of the subject.

In order to evaluate the mechanisms of AWER-and memorization processes under anesthesia-we hope that a multidisciplinary approach could be applied to this issue through (a) ad hoc studies on humans under anesthesia; (b) regression therapy studies after anesthesia, and (b) studies in animal models.

Studies on humans under anesthesia. Implicit memory is often studied experimentally through the use of priming procedures in which the subject is first exposed to a brief or degraded stimulus (e.g., few words) and after an interval ranging from minutes to months, the participant is then exposed to an incomplete stimulus, such as a word fragment, and is asked to recognize or identify it. If the previous exposure to the prime facilitates the identification of the stimulus and the completion of the task, then the subject must have some implicit memory for it. Priming approaches are used to train a subject's memory both in positive and negative ways (i.e., positive and negative primings), for instance in elderly [22].

The isolated forearm technique (IFT) could be helpful for this purpose for performing the first part of the procedure. A positive result after completing the procedure could be indicative of memory consolidation process under anesthesia without any type of recall. The IFT is usually performed by putting a cuff on the patient's arm before the administering of the neuromuscular blocking drug, so that the patient is able to move the arm, if aware [23]. Surprisingly, many of the 'responder' patients under anesthesia do not recall the event when interviewing after the operation. This evidence leads to two considerations. First, unrecognized episodes of consciousness may occur during anesthesia and not be reported upon awakening, due to a lack of explicit consolidation. In this way, patients may process information whilst only apparently unconscious. We should, therefore, assume that during anesthesia episodes of consciousness fluctuation may occur [16]. Secondly, a great number of pieces of information (i.e., unconscious experiences) may be consolidated via implicit memorization processes. In other words, we must investigate in order to decide if these IA episodes may be defined as conscious experiences without subsequent recollection on recovery due to the amnesic effects of anesthetics or general forgetting, or they really represent the expression of unconscious experiences. Probably, both hypotheses could be accepted. Regardless of the precise mechanism, this dangerous implicit memory formation might be difficult to avoid, triggering a dangerous cascade which ends in behavior changes, and potentially in psychological trauma. Thus, IA prevention is mandatory. For this purpose, the combination of typical clinical signs with instrumental monitoring is the standard approach. However, despite initial enthusiasm, the use of EEG based depth of anesthesia (DoA) monitors (e.g., bispectral, BIS) remains a major controversy in anesthesiology, and routine use of DOA devices did not reduce the overall IA incidence [24]. Indeed, many factors, such as age, race, gender, low core body temperature, acid-base imbalances, low blood glucose, drugs administered to the patient (e.g. neuromuscular blocking agents), and brain ischemia, have a significant effect on raw EEG data. Again, a limit in brain monitoring during anesthesia is that commercial 
DOA devices do not accurately discriminate between consciousness and unconsciousness because their functioning is based on EEG signal analysis rather than consideration of corticocortical connectivity and communication [25].

Whether the use of IFT approach, the optimal strategy for studying AWER processes is the implicit memory assessment by a word memory test administered during anesthesia. The assumption is that auditory processing persists when people are anesthetized, in the absence of consciousness. A special issue concerns the selection of the more appropriate memory tests for priming during anesthesia. For this scope, several attempts have been performed by using perceptual or conceptual priming tests. The former relates to the stimuli's form and is increased by matches between early and late stimuli. An example of this is the completion of words in the aforementioned word-stem completion test. Conceptual priming refers to the meaning of stimuli. Thus, this latter approach is based on the conceptual implicit memory which requires attentional resources [26]. Perceptual priming is preferred for studying memory under anesthesia because it is more suitable for assessing memory in the absence of conscious awareness $[27,28]$.

Studies under regression therapy. Regression therapy is a psychotherapeutic methodology based on resolving significant past events believed to be interfering with a person's present mental and emotional wellness. However, this approach is somewhat controversial, due to limited research supporting the method and the potential for false memories.

Animal models. As Jabes et al. [29] demonstrated, implicit memory in human is mainly associated with the striatum, the cerebellum, the amygdala and the sensory and motor areas. All these brain areas are phylogenetically old and develop also in nonhuman primates. The eyeblink conditioning, a cerebellum-dependent learning paradigm, is one of the best-characterized behavioral models of associative learning in mammals [30]. Alternatively, a modified version of the Active/Passive avoidance test and the Morris water maze test - both using rats - could be adopted. These models could be useful to test specific pharmacologic approaches aimed at prevention of memory consolidation during anesthesia [31].

\section{Conclusion}

Although the IA subtype AWER is an uncommon general anesthesia complication, this underestimated phenomenon is strictly closed to the development of severe postoperative psychological/ psychiatric sequelae, leaving patients depending on mental health care for an extended period after surgery. Moreover, studying into this 'paradox' offers an opportunity to investigate on memory and consciousness processes during anesthesia. Given the complexity of the matter, a multidisciplinary approach involving scientists from different fields are required.

\section{Funding and conflict of interest statement}

Both authors certify that no funding has been received for the preparation of this manuscript and declare that they have no conflict of interest.

\section{References}

1. Chung HS (2014) Awareness and recall during general anesthesia. Korean J Anesthesiol 66: 339-345. [Crossref]

2. Cascella M (2014) What about Memory, Consciousness, Recall, and Awareness in Anesthesia? Iran J Med Sci 39: 311-312. [Crossref]
3. Bailey AR, Jones JG (1997) Patients' memories of events during general anaesthesia. Anaesthesia 52: 460-76. [Crossref]

4. Cascella M, Fusco R, Caliendo D, Granata V, Carbone D, et al. (2017) Anesthetic dreaming, anesthesia awareness and patient satisfaction after deep sedation with propofol target controlled infusion: A prospective cohort study of patients undergoing day case breast surgery. Oncotarget 8: 79248-79256. [Crossref]

5. Pandit JJ, Andrade J, Bogod DG, Hitchman JM, Jonker WR, et al. (2014) The 5th National Audit Project (NAP5) on accidental awareness during general anaesthesia: summary of main findings and risk factors. Br J Anaesthesia 69: 1089-1101. [Crossref]

6. Brice DD, Hetherington RR, Utting JE (1970) A simple study of awareness and dreaming during anaesthesia. Br J Anaesth 42: 535-542. [Crossref]

7. Bischoff P, Rundshagen I, Schneider G (2015) Undesired awareness phenomena during general anesthesia: Evidence-based state of knowledge, current discussions and strategies for prevention and management. Anaesthesist 64: 732-739. [Crossref]

8. Sebel PS, Bowdle TA, Rampil IJ, Padilla RE, Gan TJ, et al. (2007) Don't ask, don't tell. Anesthesiology 107: 672. [Crossref]

9. Cascella M, Viscardi D, Schiavone V, Mehrabmi-Kermani F, Muzio MR, et al. (2016) A 7-Year Retrospective Multisource Analysis on the Incidence of Anesthesia Awareness with Recall in Cancer Patients: A Chance of Collaboration Between Anesthesiologists and Psycho-Oncologists for Awareness Detection. Medicine 95: e2757.

10. Osterman JE, van der Kolk BA (1998) Awareness during anesthesia and posttraumatic stress disorder. Gen Hosp Psychiatry 20: 274-281. [Crossref]

11. Myles PS, Leslie K, McNeil J, Forbes A, Chan MT (2004) Bispectral index monitoring to prevent awareness during anaesthesia: the B-Aware randomised controlled trial. Lancet 363: 1757-1763. [Crossref]

12. https://dictionary.cambridge.org/it/dizionario/inglese/recollection

13. Cascella M (2015) The Impact of Anesthetics Drugs on Memory and Memory Modulation under General. In Silico In Vitro Pharmacol 1: 1-6.

14. Elsey JWB, Van Ast VA, Kindt M (2018) Human memory reconsolidation: A guiding framework and critical review of the evidence. Psychol Bull.

15. Squire LR, Wixted JT (2011) The cognitive neuroscience of human memory since H.M. Аnпu Rev Neurosci 34: 259-288. [Crossref]

16. Cascella M, Schiavone V, Muzio MR, Cuomo A (2016) Consciousness fluctuation during general anesthesia: a theoretical approach to anesthesia awareness and memory modulation. Curr Med Res Opin 32: 1351-1359.

17. Veselis RA (2015) Memory formation during anaesthesia: plausibility of a neurophysiological basis. Br J Anaesth 115: i13-i19.

18. Sanders RD, Tononi G, Laureys S, Sleigh JW (2012) Unresponsiveness? unconsciousness. Anesthesiology: 116: 946-959.

19. Samuelsson P, Brudin L, Sandin RH (2007) Late psychological symptoms after awareness among consecutively included surgical patients. Anesthesiology 106: 2632.

20. Mashour GA, Avidan MS (2015) Intraoperative awareness: controversies and noncontroversies. Br J Anaesth 115: Si20-S26. [Crossref]

21. Dutton RC, Smith WD, Smith NT (1995) Brief wakeful response to command indicates wakefulness with suppression of memory formation during surgical anesthesia. $J$ Clin Monit 11: 41-46.

22. Hagood EW, Gruenewald TL (2018) Positive versus negative priming of older adults' generative value: do negative messages impair memory? Aging Ment Health 22: 257 260. [Crossref]

23. Andrade J, Deeprose C (2007) Unconscious memory formation during anaesthesia Best Pract Res Clin Anaesthesiol 21: 385-401. [Crossref]

24. Avidan MS, Zhang L, Burnside BA, Finkel KJ, Searleman AC, et al. (2008) Anesthesia awareness and the bispectral index. N Engl J Med 358: 1097-1108. [Crossref]

25. Cascella M (2016) Mechanisms underlying brain monitoring during anesthesia limitations, possible improvements, and perspectives. Korean J Anesthesiol 69: 113-120.

26. Sbicigo JB, Janczura GA, de Salles JF (2017) The role of attention in perceptual and conceptual priming. Psychology and Neuroscience 10: 117-131.

27. Aktas G, Sahin E, Turkay Aydogmus M, Erkin Y (2013) The assessment of memory under total intravenous anesthesia. Braz J Anesthesiol 63: 301-306. [Crossref]

28. Deeprose C, Andrade J (2006) Is priming during anesthesia unconscious? Conscious Cogn 15: 1-23. [Crossref]

29. Jabes A, Nelson CA (2015) 20 years after "The ontogeny of human memory: A cognitive neuroscience perspective," where are we? Int J Beh Dev 39: 293-303. 
30. Thompson RF, Krupa DJ (1994) Organization of memory traces in the mammalian brain. Annu Rev Neurosci 17: 519-549. [Crossref]
31. Cascella M (2015) Anesthesia awareness. Can midazolam attenuate or prevent memory consolidation on intraoperative awakening during general anesthesia without increasing the risk of postoperative delirium? Korean J Anesthesiol 68: 200-202. [Crossref]

Copyright: (C2018 Cascella M. This is an open-access article distributed under the terms of the Creative Commons Attribution License, which permits unrestricted use, distribution, and reproduction in any medium, provided the original author and source are credited. 